\title{
Revisiting Graph Based Collaborative Filtering: A Linear Residual Graph Convolutional Network Approach
}

\author{
Lei Chen, ${ }^{1,2}$ Le Wu, ${ }^{1,2, *}$ Richang Hong, ${ }^{1,2}$ Kun Zhang, ${ }^{3}$ Meng Wang ${ }^{1,2}$ \\ ${ }^{1}$ Key Laboratory of Knowledge Engineering with Big Data, Hefei University of Technology \\ ${ }^{2}$ School of Computer Science and Information Engineering, HeFei University of Technology \\ ${ }^{3}$ School of Computer Science and Technology, University of Science and Technology of China \\ \{chenlei.hfut, lewu.ustc, hongrc.hfut, eric.mengwang\}@gmail.com, zhkun@ mail.ustc.edu.cn
}

\begin{abstract}
Graph Convolutional Networks (GCNs) are state-of-the-art graph based representation learning models by iteratively stacking multiple layers of convolution aggregation operations and non-linear activation operations. Recently, in Collaborative Filtering (CF) based Recommender Systems (RS), by treating the user-item interaction behavior as a bipartite graph, some researchers model higher-layer collaborative signals with GCNs. These GCN based recommender models show superior performance compared to traditional works. However, these models suffer from training difficulty with non-linear activations for large user-item graphs. Besides, most GCN based models could not model deeper layers due to the over smoothing effect with the graph convolution operation. In this paper, we revisit GCN based CF models from two aspects. First, we empirically show that removing non-linearities would enhance recommendation performance, which is consistent with the theories in simple graph convolutional networks. Second, we propose a residual network structure that is specifically designed for $\mathrm{CF}$ with useritem interaction modeling, which alleviates the over smoothing problem in graph convolution aggregation operation with sparse user-item interaction data. The proposed model is a linear model and it is easy to train, scale to large datasets, and yield better efficiency and effectiveness on two real datasets. We publish the source code at https://github.com/newlei/LRGCCF.
\end{abstract}

\section{Introduction}

Recent years have witnessed the boom of GCNs, which are efficient variants of CNNs for dealing with graph based data (Kipf and Welling 2017; Hamilton, Ying, and Leskovec 2017). The key idea of GCNs is to stack multiple layers that iteratively perform the following two steps at each layer: node embedding with convolutional neighborhood aggregation; followed by a non-linear transformation of node embeddings parameterized by a neural network. Therefore, the higher-order similarity of a node could be effectively captured (Xu et al. 2019b; Li, Han, and Wu 2018). These models show competing performance for tasks such as unsupervised

\footnotetext{
${ }^{*}$ Corresponding Author Copyright (c) 2020, Association for the Advancement of Artificial Intelligence (www.aaai.org). All rights reserved.
}

node (graph) representation learning (Li et al. 2016), semisupervised node (graph) learning (Kipf and Welling 2017; Camps-Valls, Marsheva, and Zhou 2007), and so on.

As many real-world data show the graph structure, GCNs have been widely applied to applications such as social network analysis (Xu et al. 2019a), transportation network (Zhao et al. 2019), and recommender systems (Berg, Kipf, and Welling 2018). In this paper, we focus on applying GCNs to CF based recommender systems. CF provides personalized item suggestions to users by learning user and item embeddings from their historical behavior data (Rendle et al. 2009; He et al. 2017). In fact, by treating the user-item historical behavior as a bipartite graph with edges between users and items, CF can be naturally transformed into the edge prediction problem in the graph. This graph representation of user-item behavior advances previous user-item 6 interaction matrix with more higher-order user and item correlations, and provides the possibility to alleviate the data sparsity issue in $\mathrm{CF}$ with graph structure modeling (Wang et al. 2019; Berg, Kipf, and Welling 2018; Ying et al. 2018). Some earlier works applied personalized random walks (Liu and Yang 2008) or relied on graph regularization models with auxiliary graph data (e.g., social network) for recommendation (Gu, Zhou, and Ding 2010; Huang, Chung, and Chen 2004). These models suffered from a huge time complexity with personalized random walk, and most of these models relied on carefully designing the random walk process. Recently, plenty of researchers pay more attention to apply GCNs for recommendation (Wu, Liu, and Yang 2018; Wang et al. 2019; Berg, Kipf, and Welling 2018; Ying et al. 2018). For example, PinSage designed sampling techniques for graph convolution aggregation to alleviate the computational burden in the recommendation process (Ying et al. 2018). By feeding the user and item free embeddings as input, NGCF was specially designed for GCN based CF (Wang et al. 2019). NGCF iteratively propagates user and item embeddings in the graph to distill the collaborative signals with graph convolutions. These GCN based recommender models show better performance compared to traditional models.

Despite the relative success of GCN based recommendation, we argue that two important problems in GCN based 
$\mathrm{CF}$ still remain unsolved. On one hand, for user and item embeddings, GCNs follow the two steps of neighborhood aggregation with graph convolutional operations and nonlinear transformations. While graph convolutional operations are effective for aggregating the neighborhood information and modeling higher order graph structure, is the additional complexity introduced by the non-linear feature transformation in GCNs necessary? On the other hand, most of the current GCN based models could only stack very few layers (e.g., 2 layers). In fact, the graph convolution operation is a special kind of Graph Laplacian smoothing (Li, Han, and Wu 2018; Klicpera, Bojchevski, and Günnemann 2019). With $K$-th layer of GCNs, the Laplacian smoothing is performed to incorporate the up to $K$-th neighbors. Therefore, the over-smoothing effect exists with deep layers, as the higher layer neighbors tend to be indistinguishable for each node. With limited user-item interaction records in the recommendation (Wu et al. 2017; 2016), this problem would become more severe since the training records are very sparse. Intuitively, with the increasing of the stacking layers, the smoothing effect could alleviate the data sparsity of CF at first, but the over smoothing effect introduced by more layers would neglect each user's uniqueness and degrade the recommendation performance. How to better model the graph structure while avoiding the over smoothing effect in this process remains pretty much open.

To tackle the above two issues, we revisit the graph based $\mathrm{CF}$ models with a linear residual graph convolutional approach. Our main contributions lie in two aspects: on one hand, we empirically analyze the uniqueness of $\mathrm{CF}$ from most graph based tasks, and show that removing the nonlinearity would enhance the recommendation performance with less complexity, which is consistent with the recent theories in simplifying GCNs (Wu et al. 2019a). Furthermore, to alleviate the over smoothing problem in the iterative process, we propose to learn the residual user-item preference at each layer. Thus, the user uniqueness is preserved at the lower layers, while the higher layers of the GCNs could focus on learning users' residual preferences that could not be captured from each user's limited historical records. Please note that this idea is inspired the ResNet architecture in CNNs (He et al. 2016; Wu, Shen, and Van Den Hengel 2019), and our work focuses on how to extend the formulation of the residual part in $\mathrm{CF}$ with the interaction prediction between users and items under GCNs. We then show that with linear residual learning, our proposed model degenerates to a linear model that effectively leverages the user-item graph structure for recommendation. In summary, in contrast to current GCN based recommendation models, our proposed model is easier to train, scales to large datasets. Finally, we perform extensive experiments on two large real-world CF datasets, and the results clearly show the effectiveness and efficiency of our proposed model.

\section{Preliminaries and Related Work}

Considering a graph $\mathcal{G}=\langle\mathcal{V}, \mathbf{A}\rangle$, with $\mathcal{V}$ is the set of nodes and $\mathbf{A}$ is the adjacency matrix, in which $a_{i j}$ denotes the edge between node $i$ and node $j$. If there is a directed edge from node $i$ to node $j$, then $a_{i j}=1$, otherwise it is 0 . For ease of notation, we use $S_{i}=\left[j \mid a_{i j}=1\right]$ to denote the neighbor set of node $i_{2}$ i.e., the node set that $i$ connects to. We use $\mathbf{S}=$ $\tilde{\mathbf{D}}^{-0.5} \tilde{\mathbf{A}} \tilde{D}^{-0.5}$ to denote the normalized adjacency matrix with added self loops, with $\tilde{\mathbf{A}}=\mathbf{A}+\mathbf{I}$ is the adjacency matrix of the graph with added self-connections, and $\mathbf{I}$ is the identity matrix. $\tilde{\mathbf{D}}$ is the degree matrix of $\tilde{\mathbf{A}}$.

\section{Graph Convolutional Networks}

For each node $i \in \mathcal{V}$, we use $\mathbf{h}_{i}^{0}$ to denote the node initial embedding, which is usually the feature vector $\mathbf{x}_{i}$ of node $i$ (i.e, $\mathbf{h}^{0}=\mathbf{x}_{i}$ ). In a graph $\mathcal{G}$, the key idea of GCNs is to stack $K$ steps in a recursive message passing or feature propagation manner to learn node embeddings (Berg, Kipf, and Welling 2018; Hamilton, Ying, and Leskovec 2017; Gilmer et al. 2017). Specifically, for each node $i$ at the step, it is computed recursively with following two steps: feature propagation and non-linear feature transformation.

Feature propagation. For each node $i$, the feature aggregation step aggregates the embeddings from graph neighbors $S_{i}$ and its own embedding $\mathbf{h}_{i}^{k}$ at previous layer $k$.

Earlier works focus on how to model the aggregation functions (Hamilton, Ying, and Leskovec 2017; Gilmer et al. 2017; Velickovic et al. 2018; Kipf and Welling 2017). As the focus of this paper is not to design more sophisticated feature aggregation function, we follow the widely used feature aggregation function proposed in Kipf et al. (Kipf and Welling 2017), which is empirically effective and has been adopted by many GCN variants (Kipf and Welling 2017; Berg, Kipf, and Welling 2018; Wu et al. 2019a):

$$
\overline{\mathbf{H}}^{(k+1)}=\tilde{\mathbf{D}}^{-0.5} \tilde{\mathbf{A}} \tilde{\mathbf{D}}^{-0.5} \mathbf{H}^{k} .
$$

In fact, given the features $\mathbf{H}^{k}$ at $k$-th layer, feature propagation output $\overline{\mathbf{H}}^{(k+1)}$ layer can be regarded as the Laplacian smoothing on the features at the previous layer ( $\mathrm{Li}, \mathrm{Han}$, and Wu 2018; Zhu, Ghahramani, and Lafferty 2003).

Nonlinear transformation. The nonlinear transformation layer is a standard Multilayer Perceptron (MLP). By feeding the output of the feature propagation step, the nonlinear transformation produces the $(k+1)$-th layer embedding of each node as:

$$
\mathbf{H}^{(k+1)}=\sigma\left(\overline{\mathbf{H}}^{(k+1)} \mathbf{W}^{k}\right),
$$

where $\sigma(x)$ is a non-linear activation function.

After iteratively performing the two steps in each layer with a defined depth $K$, the final embedding of each node at depth $K$ is $\mathbf{h}_{i}^{K}$. For most GCN based applications, there is a prediction function $f($.$) as:$

$$
\hat{y}=f\left(\mathbf{h}_{i}^{K} \mid i \in V\right) .
$$

As GCNs derive inspiration primarily from the CNNs in the deep learning community, it inherits considerable nonlinearity and complexity from the nonlinear transformations as shown in Eq.(2). Researchers exploit the possibility of simplifying GCNs. Recently, a Simple Graph Convolution (SGC) is proposed (Wu et al. 2019a), which removes the nonlinear transformation in Eq.(2) as: 


$$
\mathbf{H}^{K}=\mathbf{S S} \ldots \mathbf{S H}^{\mathbf{0}} \mathbf{W}^{\mathbf{0}} \mathbf{W}^{\mathbf{1}} \ldots \mathbf{W}^{\mathbf{K}},
$$

where we can rewrite $\mathbf{W}^{0} \mathbf{W}^{1} \ldots \mathbf{W}^{K}$ as a single matrix $\mathbf{W}$, and the above linear matrix multiplication turns to:

$$
\mathbf{H}^{K}=\mathbf{S S} \ldots \mathbf{S H}^{0} \mathbf{W} .
$$

With the formulation of SGC, GCNs reduce to the iterative simple feature propagations with very few parameters. Therefore, it is easy to tune and scales to large datasets. As verified by researchers, SGCN corresponds to a fixed low pass filter on graph spectral domain. Besides, the empirical evaluations show that SGCN does not negatively impact accuracy in many graph based tasks with huge time improvement (Wu et al. 2019a).

\section{Graph Convolutional based Recommendation}

In a recommender system, there are two sets of entities: a userset $\mathcal{U}$ with $M$ users $(|\mathcal{U}|=M)$ and an itemset $\mathcal{V}(|\mathcal{V}|=N)$. As implicit feedback is the most common form in many recommender systems, we focus on implicit feedback based CF in this paper (Rendle et al. 2009), and it is easy to extend the proposed model for rating prediction in CF. Users show ratings to the items with a rating matrix $\mathbf{R} \in \mathbb{R}^{M \times N}$, with $r_{a i}=1$ denotes user $a$ likes item $i$, otherwise it equals 0 . With the rating matrix, accurately learning user embedding matrix and item embedding matrix is a key to the success of recommendation performance. Earlier works focus on shallow matrix factorization based models (Koren, Bell, and Volinsky 2009; Rendle et al. 2009). Deep learning based models, e.g., NeuMF (He et al. 2017), and Wide\&Deep (Cheng et al. 2016) modeled the interaction between users and items with a deep neural network structure.

With the huge success of GCNs, researchers attempted to formulate recommendation as a user-item bipartite graph, and adapted GCNs for recommendation (Wang et al. 2019; Monti, Bronstein, and Bresson 2017; Ying et al. 2018). Earlier works on GCN based models relied on the spectral theories of graphs, and are computationally costly when applying in real-world recommendations (Monti, Bronstein, and Bresson 2017; Zheng et al. 2018). Some of recent works on GCN based recommendation models focused on the spatial domain (Wu, Liu, and Yang 2018; Wang et al. 2019; Berg, Kipf, and Welling 2018; Ying et al. 2018). PinSage was designed for similar item recommendation under the content based model, with the item features $\mathbf{x}_{v}$ and the itemitem correlation graph as the inputs (Ying et al. 2018). GCMC (Berg, Kipf, and Welling 2018) and NGCF (Wang et al. 2019) are specifically designed under the CF setting. Given ratings of users to items, the user-item bipartite graph is denoted as $\mathcal{G}=\langle\mathcal{U} \cup \mathcal{V}, \mathbf{A}\rangle$, with $\mathbf{A}$ is constructed from the rating matrix $\mathbf{R}$ as:

$$
\mathbf{A}=\left[\begin{array}{cc}
\mathbf{R} & \mathbf{0}^{N \times M} \\
\mathbf{0}^{M \times N} & \mathbf{R}^{\mathbf{T}}
\end{array}\right] .
$$

Let $\mathbf{E} \in \mathbb{R}^{(M+N) \times D}$ denote the free embedding matrix of users and items. By feeding the free embedding matrix $\mathbf{E}$ into GCNs with bipartite graph $\mathcal{G}$, i.e., $\forall i \in \mathcal{U} \cup \mathcal{V}, \mathbf{h}_{i}^{0}=\mathbf{e}_{i}$.
Then, GCNs iteratively perform with embedding propagation step in Eq.(1) and nonlinear transformation with Eq.(2) and each user's (item's) embeddings can be updated in the iterative process. Therefore, the final embedding $\mathbf{H}^{K}$ explicitly injects the up to $K$-th order collective connections between users and items. All the parameters (including the initial free embedding matrix $\mathbf{E}$, the transformation parameters $\left.\left(\left[\mathbf{W}^{k}\right]_{k=0}^{K}\right)\right)$ can be learned in an end-to-end manner. GC-MC could be seen a special case of NGCF with $K=1$, i.e., only the first order connectivity of the user-item bipartite graph is modeled (Berg, Kipf, and Welling 2018).

\section{Deep Network Architecture Design}

Theoretically, deep neural networks could approximate complex functions (Goodfellow, Bengio, and Courville 2016). However, many researchers found stacking deeper layers in the network usually would not correspondingly increase performance in practice. For example, in the computer vision domain, directly stacking more layers in CNNs would complex the model training process, which leads to degradation of the image classification performance. For example, many CNNs variants have been proposed to how to stack more deep layers to improve image classification performance. (He et al. 2016; Huang et al. 2017). Researchers argued that the degradation of the deeper layers in CNNs is not caused by overfitting, but the harder training process with higher training error compared to the relatively shallower models. Therefore, a deep residual learning framework, i.e., ResNet, is proposed to reformulate the layers as learning residual functions, which is easier to train compared to directly learning original functions (He et al. 2016). In CF based recommender systems, simply relying on the deep neural networks would also not perform well due to the sparseness of user behavior data. Therefore, many deep learning based CF models have two parts: a shallow wide part and a deep neural network part, such as NeuMF (He et al. 2017) and Wide\&Deep (Cheng et al. 2016). The deep architecture design problem also exists in GCN variants. For example, many GCN based models achieve the best performance with layer depth of 2 (Hamilton, Ying, and Leskovec 2017; Wu et al. 2019b). As the local network structure varies from node to node, researchers proposed to aggregate all layer representations at the last layer (Xu et al. 2018), or allowed the root node teleport to the later layers (Klicpera, Bojchevski, and Günnemann 2019). In order to overcome the limitations of GCN models with limited labeled data, cotraining and self-training approaches are proposed to train GCNs to supplement sparse labeled data ( $\mathrm{Li}, \mathrm{Han}$, and $\mathrm{Wu}$ 2018). We differ from these works on two aspects. First, our model is based on a GCN with linear structure compared to these nonlinear GCNs. Moreover, our proposed architecture is concerned with how to better preserve the previous layer information with a residual network structure. 


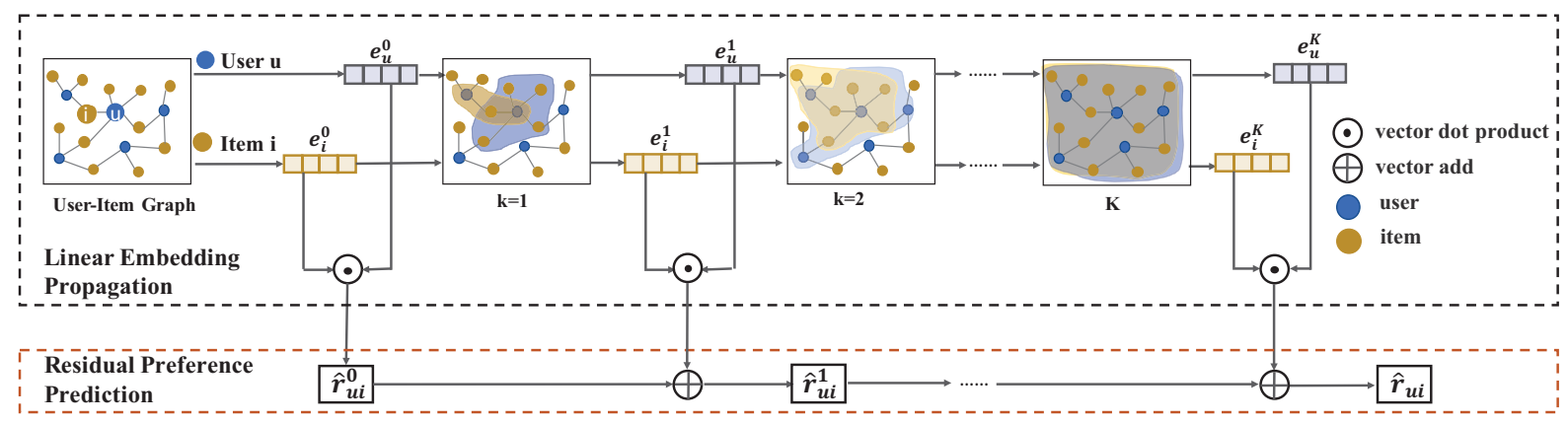

Figure 1: The overall architecture of our proposed model.

\section{Linear Residual Graph Convolutional Collaborative Filtering}

\section{Overall Structure of the Proposed Model}

In this part, we propose Linear Residual Graph Convolutional Collaborative Filtering (LR-GCCF) which is a general GCN based CF model for recommendation. The overall architecture of LR-GCCF is shown in Figure 1. LR-GCCF advances current GCN based models with two characteristics: (1) At each layer of the feature propagation step, we use a simple linear embedding propagation without any nonlinear transformations. (2) For predicting users' preferences of items, we propose a residual based network structure to overcome the limitations of previous works.

Linear Embedding Propagation Given the user-item bipartite graph as formulated in Eq.(6), let $\mathbf{E} \in \mathbb{R}^{(M+N) \times D}$ denotes the free embeddings of users and items, with the first $M$ rows of the matrix, i.e., $\mathbf{E}_{[1: M]}$ is the user embedding submatrix, and $\mathbf{E}_{[M+1: M+N]}$ is the item embedding submatrix. Then, LR-GCCF takes the embedding matrix as input:

$$
\mathbf{E}^{0}=\mathbf{E}
$$

which resembles the embedding based models in CF. Notably, different from GCN based tasks with node features as fixed input data, the embedding matrix is unknown and needs to be trained in LR-GCCF.

Following the theoretical elegance with graph spectral connections and empirical competing results of SGCN (Wu et al. 2019a), at each iteration step $k+1$, we assume the embedding matrix $\mathbf{E}^{(k+1)}$ is a linear aggregation of the embedding matrix $\mathbf{E}^{k}$ at the previous layer $k$ as:

$$
\mathbf{E}^{k+1}=\mathbf{S E}^{k} \mathbf{W}^{k}
$$

where $\mathbf{S}=\tilde{\mathbf{D}}^{-0.5} \tilde{\mathbf{A}} \tilde{\mathbf{D}}^{-0.5}$ denotes the normalized adjacency matrix with added self loops, $\mathbf{W}^{k}$ is the linear transformation.

In fact, Eq.(8) with matrix form is equivalent to modeling each user $u$ 's and each item $i$ 's updated embedding as:

$$
\begin{aligned}
{\left[\mathbf{E}^{k+1}\right]_{u} } & =\mathbf{e}_{u}^{k+1}=\left[\frac{1}{d_{u}} \mathbf{e}_{u}^{k}+\sum_{j \in R_{u}} \frac{1}{d_{j} \times d_{u}} \mathbf{e}_{j}^{k}\right] \mathbf{W}^{k} \\
{\left[\mathbf{E}^{k+1}\right]_{i} } & =\mathbf{e}_{i}^{k+1}=\left[\frac{1}{d_{i}} \mathbf{e}_{i}^{k}+\sum_{u \in R_{i}} \frac{1}{d_{i} \times d_{u}} \mathbf{e}_{u}^{k}\right] \mathbf{W}^{k},
\end{aligned}
$$

which $d_{i}\left(d_{u}\right)$ is the diagonal degree of item $i$ (user $u$ ) in the user-item bipartite graph $\mathcal{G} . R_{*}$ is neighbors of node $(*)$ in graph $\mathcal{G}$.

Residual Preference Prediction With a predefined depth $K$, the recursive linear embedding propagation would stop at the $K$-th layer with output of the embedding matrix $\mathbf{E}^{K}$. For each user (item), $\mathbf{e}_{u}^{K}\left(\mathbf{e}_{i}^{K}\right)$ captures the up to K-th order bipartite graph similarity. Then, many embedding based recommendation models would predict the preference $\hat{r}_{u i}$ as the inner product between user and item latent vectors as:

$$
\hat{r}_{u i}=<\mathbf{e}_{u}^{K}, \mathbf{e}_{i}^{K}>\text {, }
$$

where $<,>$ denotes vector inner product operation.

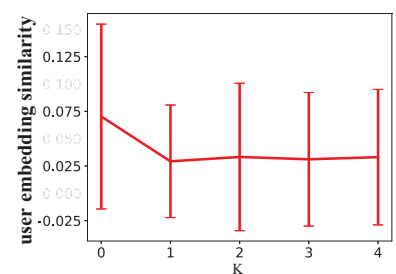

(a) Errorbar of user embedding similarity

\begin{tabular}{|l|l|l|l|l|l|}
\hline Depth & 0 & 1 & 2 & 3 & 4 \\
\hline NDCG@20 & 0.0217 & $\mathbf{0 . 0 2 2 4}$ & $\mathbf{0 . 0 2 4 2}$ & $\mathbf{0 . 0 2 4 2}$ & $\mathbf{0 . 0 2 4 1}$ \\
\hline
\end{tabular}
(c) Overall performance on recommendation performance

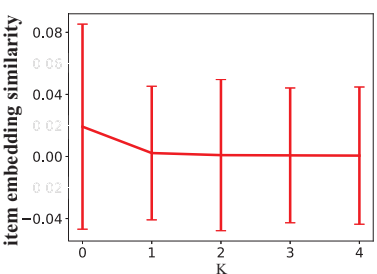

(b) Errorbar of item embedding similarity
Figure 2: GCN based recommendation performance with different layers $K$ on Amazon Books dataset.

In practice, most GCN based variants, as well as GCN based recommendation models, achieve the best performance with $K=2$ (Kipf and Welling 2017; Hamilton, Ying, and Leskovec 2017; Ying et al. 2018). The overall trend for these GCN variants is that: the performance increases as $K$ increases from 0 to 1 (2), and drops quickly as $K$ continues to increase, the performance drops quickly. We speculate a possible reason is that, at the $k$-th layer, the embedding of each node is smoothed by the k-th order neighbors in the bipartite graph. Therefore, as $k$ increases from 0 to $K$, the node embeddings at deeper layers tend to be over smoothed, i.e., they are more similar with less distinctive information. This problem not only exists in GCNs, but is 
much more severe in $\mathrm{CF}$ with very sparse user behavior data for model learning. To validate the over assumption, we show the performance of GCN based recommendation with user-item bipartite graph using the predicted function in Eq.(11) with different depth $K$. When $K=0$, the GCN based recommendation model degenerates to BPR (Rendle et al. 2009). To empirically show the over smoothing hypothesis, with each value of $K$, we calculate the average pair-wise user-user (item-item) embedding similarity with cosine similarity at the $K$-th layer output. Specifically, for each pair of user $a$ and user $b$, their similarity is calculated as: $\operatorname{sim}(a, b)=\frac{<\mathbf{e}_{a}^{K}, \mathbf{e}_{b}^{K}>}{\left\|\mathbf{e}_{a}^{K}\right\|^{2},\left\|\mathbf{e}_{b}^{K}\right\|^{2}}$. Then, we plot the mean and variance of the cosine similarity of all pairs in Figure 2, with the recommendation performance is listed at the bottom. We have two observations from this figure. First, the variance between user (item) embeddings are smaller when $K$ increases, due to the fact of the up to $K$-th order smoothness with neighborhood regularization. Second, when $K=0$, the recommendation performance is rather good. As we increase $K$ from 0 to 2 , the performance increases less than $10 \%$. Therefore, we empirically conclude that BPR $(K=0)$ could already approximate preference of user to a large extent.

Based on the above two observations, we argue that: instead of directly approximating the user preference of each user-item pair at each layer, we perform the residual preference learning as:

$$
\hat{r}_{u i}^{k+1}=\hat{r}_{u i}^{k}+<\mathbf{e}_{u}^{k+1}, \mathbf{e}_{i}^{k+1}>.
$$

We hypothesis that it is easier to optimize the residual rating than to optimize the original rating, and the residual learning could help to alleviate the over smoothing effect with deeper layers.

Based on the residual preference prediction in above Eq.(12), we have:

$$
\begin{aligned}
\hat{r}_{u i} & =\hat{r}_{u i}^{K-1}+<\mathbf{e}_{u}^{K}, \mathbf{e}_{i}^{K}> \\
& =\hat{r}_{u i}^{K-2}+<\mathbf{e}_{u}^{K-1}, \mathbf{e}_{i}^{K-1}>+<\mathbf{e}_{u}^{K}, \mathbf{e}_{i}^{K}> \\
& =\hat{r}_{u i}^{0}+<\mathbf{e}_{u}^{1}, \mathbf{e}_{i}^{1}>+\ldots+<\mathbf{e}_{u}^{K}, \mathbf{e}_{i}^{K}> \\
& =<\mathbf{e}_{u}^{0}\left\|\mathbf{e}_{u}^{1}\right\| \ldots\left\|\mathbf{e}_{u}^{K}, \quad \mathbf{e}_{i}^{0}\right\| \mathbf{e}_{i}^{1}\|\ldots\| \mathbf{e}_{i}^{K}>.
\end{aligned}
$$

The above equation is equivalent to concatenate embedding of each layer to form the final embedding of each node. This is quite reasonable as each node's sub-graph varies, and recording each layer's representation to form the final embedding of each node is more informative.

Model Learning By putting the linear embedding propagation equation (Eq.(8)) into vector representation of the residual prediction function (Eq.(13)), we have:

$$
\begin{aligned}
\hat{r}_{u i}=< & \mathbf{e}_{u}^{0}\left\|\mathbf{e}_{u}^{1}\right\| \ldots\left\|\mathbf{e}_{u}^{K}, \quad \mathbf{e}_{v}^{0}\right\| \mathbf{e}_{v}^{1}\|\ldots\| \mathbf{e}_{v}^{K}> \\
=< & {\left[\mathbf{E}^{0}\right]_{u}\|\ldots\|\left[\mathbf{S}^{K} \mathbf{E}^{0} \mathbf{W}^{0} \ldots \mathbf{W}^{K}\right]_{u} } \\
& {\left[\mathbf{E}^{0}\right]_{i}\|\ldots\|\left[\mathbf{S}^{K} \mathbf{E}^{0} \mathbf{W}^{0} \ldots \mathbf{W}^{K}\right]_{i}>} \\
=< & {\left[\mathbf{E}^{0}\right]_{u}\|\ldots\|\left[\mathbf{S}^{K} \mathbf{E}^{0} \mathbf{Y}^{K}\right]_{u}, \quad\left[\mathbf{E}^{0}\right]_{i}\|\ldots\|\left[\mathbf{S}^{K} \mathbf{E}^{0} \mathbf{Y}^{K}\right]_{i}>}
\end{aligned}
$$

where $\mathbf{Y}^{K}$ is reparameterized as $\mathbf{Y}^{K}=\mathbf{W}^{0} \mathbf{W}^{1} \ldots \mathbf{W}^{K}$ with linear multiplication. $\mathbf{S}^{K}$ denotes the $K$-th power of $\mathbf{S}$.
Since we focus on implicit feedbacks, we adopt the pairwise ranking based loss function in BPR as:

$\min _{\Theta} \mathcal{L}(\mathbf{R}, \hat{\mathbf{R}})=\sum_{a=1}^{M} \sum_{(i, j) \in D_{a}}-\ln \left(s\left(\hat{r}_{a i}-\hat{r}_{a j}\right)\right)+\lambda\left\|\Theta_{1}\right\|^{2}$,

where $s(x)$ is a sigmoid function. $\Theta=\left[\Theta_{1}, \Theta_{2}\right]$, with $\Theta_{1}=$ $\left[\mathbf{E}^{0}\right]$, and $\Theta_{2}=\left[\left[\mathbf{Y}^{k}\right]_{k=1}^{K}\right] . \lambda$ is a regularization parameter that controls the complexity of user and item free embedding matrices. $D_{a}=\left\{(i, j) \mid i \in R_{a} \wedge j \in V-R_{a}\right\}$ denotes the pairwise training data for $a$ with $R_{a}$ represents the itemset that $a$ positively shows feedback.

\section{Model Discussion}

Detailed Analysis of The Proposed Model. Based on the prediction function in Eq.(14), we observe that LR-GCCF is not a deep neural network but a wide linear model. The linearization has several advantages: First, as LR-GCCF is built on the recent progress of SGC (Wu et al. 2019a), it is theoretically connected as a low pass filter of graph on the spectral domain. Second, with the linear embedding propagation and residual preference learning, LR-GCCF is much easier to train compared to nonlinear GCN based models. Last but not least, as our model does not have any hidden layers compared to deep learning based models, we do not need back propagation training algorithms. Instead, we could resort to the stochastic gradient descent for model learning. Therefore, LR-GCCF is much more time efficient compared to classical GCN based models.

Table 1: Comparisons of different graph based recommendation models.

\begin{tabular}{|l|l|l|l|l|}
\hline \multirow{2}{*}{ Model } & \multicolumn{2}{|c|}{ Graph Structure } & \multicolumn{2}{c|}{ Model Property } \\
\cline { 2 - 5 } & $\begin{array}{l}\text { First } \\
\text { order }\end{array}$ & $\begin{array}{l}\text { Higher } \\
\text { order }\end{array}$ & $\begin{array}{l}\text { Linear } \\
\text { Propagation }\end{array}$ & $\begin{array}{l}\text { Residual } \\
\text { Prediction }\end{array}$ \\
\hline GC-MC & $\sqrt{ }$ & $\times$ & $\times$ & $\times$ \\
\hline Pinsage & $\sqrt{ }$ & $\sqrt{ }$ & $\times$ & $\times$ \\
\hline NGCF & $\sqrt{ }$ & $\sqrt{ }$ & $\times$ & $\sqrt{ }$ \\
\hline LR-GCCF & $\sqrt{ }$ & $\sqrt{ }$ & $\sqrt{ }$ & $\sqrt{ }$ \\
\hline
\end{tabular}

Connections with Previous Works. We compare the key characteristics of our proposed model with three closely related GCN based recommendation models: GC-MC (Berg, Kipf, and Welling 2018), PinSage (Ying et al. 2018), and NGCF (Wang et al. 2019). In Table 1, NGCF is one of the first few attempts that also uses a residual prediction function by taking each user (item)'s embedding as a concatenation of all layers' embeddings. However, the authors simply use this "trick" without any detailed explanation. We empirically show the reason why taking the output of the last layer embedding fails for $\mathrm{CF}$, and shows using residual prediction is equivalent to concatenate all the layer's embeddings as the final embedding of each node in the user-item bipartite graph. For PinSage, it has lower time complexity compared to its deep learning based counterparts (e.g., GC-MC and NGCF) as this model designed a sampling technique in feature aggregation process. 


\section{Experiments}

\section{Experimental Setup}

Datasets. We conduct experiments on two publicly available datasets: Amazon Books ${ }^{1}$ and Gowalla (Liang et al. 2016). We summarize the statistics of two datasets in Table 2. In data preprocessing step, we remove users (items) that have less than 10 interaction records. After that, we randomly select $80 \%$ of the records for training, $10 \%$ for validation and the remaining $10 \%$ for test.

Table 2: The statistics of the datasets.

\begin{tabular}{|l|c|c|c|c|}
\hline Dataset & Users & Items & Ratings & Rating Density \\
\hline Amazon Books & 52,643 & 91,599 & $2,984,108$ & $0.062 \%$ \\
\hline Gowalla & 29,859 & 40,981 & $1,027,370$ & $0.084 \%$ \\
\hline
\end{tabular}

Evaluation Metrics and Baselines. Since we focus on recommending items to users, we use two widely adopted ranking metrics for top- $\mathrm{N}$ recommendation evaluation: HR@N and NDCG@N (Chen et al. 2017). For each user, we select all unrated items as the negative items and combine them with the positive items the user likes in the ranking process. We compare our proposed LR-GCCF model with various state-of-the-art baselines, including the classical model BPR (Rendle et al. 2009), three graph convolutional based recommendation models: GC-MC (van den Berg, Kipf, and Welling 2017), PinSage (Ying et al. 2018), and NGCF (Wang et al. 2019). NGCF differs from PinSage as it adopts the residual learning process. Besides, in order to better verify the effectiveness of the linear and the residual learning part, we design two variants of the GC-MC: Linear-GC-MC (L-GC-MC), and Resudial-GC-MC (R-GC$\mathrm{MC}$ ), with $L$ denotes replacing the original non-linear transformation with linear embedding propagation, and $R$ denotes the preference prediction. For the baseline of NGCF, as illustrated in Table1, it adopts the residual preference learning, and when varying the non-linear embedding propagation to linear propagation, i.e., L-NGCF is the same as LRGCCF, so we do not design variants of NGCF. For our proposed model LR-GCCF, we design a simplified version of Linear-GCCF (L-GCCF). In L-GCCF, we remove the residual learning process.

Parameter Settings. We implement our LR-GCCF model in Pytorch. There are two important parameters in our proposed model: the dimension $\mathrm{D}$ of the user and item embedding matrix $\mathbf{E}$, and the regularization parameter $\lambda$ in the objective function (Eq.15). The embedding size is fixed to 64 for all models. In our proposed LRGCCF model, we try the regularization parameter $\lambda$ in the range $[0.0001,0.001,0.01,0.1]$, and find $\lambda=0.01$ reaches the best performance. We initialize the model parameters with a Gaussian distribution of mean 0 and standard deviation 0.01 . There are several parameters in the baselines, for fair comparison, all the parameters in the baselines are also tuned to achieve the best performance. For our proposed model, we empirically find that $\mathbf{Y}$ equals the identity matrix, i.e., each parameter in $\Theta_{2}$ is not learned but directly set as the identity matrix reaches the best performance.

\footnotetext{
${ }^{1}$ http://jmcauley.ucsd.edu/data/amazon/index.html
}

\section{Overall Comparison}

Table 3 and Table 4 report the overall performance comparison results on HR@N and NDCG@N. GC-MC, PinSage, and NGCF improve over BPR by leveraging the useritem bipartite graph information. In particular, GC-MC and PinSage show the effectiveness of modeling the information passing of a graph. NGCF is the baseline that captures higher-order user-item bipartite graph structure. It performs better than most baselines. Our proposed LR-GCCF model consistently outperforms NGCF, thus showing the effectiveness of modeling the user preference by the residual preference prediction and the linear embedding propagation.

In our proposed LR-GCCF, the linear embedding propagation and residual preference learning are essential parts. To gain the effectiveness of these parts, we study the performance of the variants of baselines and our simplified model of L-GC-MC. We first analyze the performance of the linear embedding propagation by comparing the linear embedding based models with the counterparts that use non-linear embeddings, i.e., L-GC-MC vs. GC-MC. We find L-GC-MC outperforms GC-MC to a large margin, and similar trends exist when comparing LR-GCCF and NGCF, empirically showing the effectiveness of the linear embedding propagation compared to the non-linear embedding propagation for GCN based recommendations. Next, we compare the performance of residual learning by comparing the results of R-GC-MC vs GC-MC, the results of NGCF vs. PinSage, and the results of LR-GCCF and L-GCCF. R-GC-MC does not show comparable performance as GC-MC, we guess a possible reason is that GC-MC is based on the first-order neighborhood aggregation. For the first-order neighborhood, each neighbor has limited neighbors and the over smoothing effect does not apply with first-order neighbors. With deep layers, the over smoothing effect becomes more severe. Thus, NGCF outperforms PinSage, and LR-GCCF outperforms L-GCCF when modeling higher-order graph structure with residual learning. Last but not least, by combing the linear propagation and the residual learning together in LRGCCF, the proposed model outperforms all the remaining models, showing the effectiveness of fusing these two parts for $\mathrm{CF}$.

Instead of the nonlinear transformation of feature propagation, our work differs from these works in a linearization method to accelerate the training process at the same time. In practice, we find that LR-GCCF is very easy to train. On Amazon Books dataset, with the best depth $K$ for each graph based recommendation model, at each iteration, the average runtime is about 30 s for GC-MC $(K=1)$, and $38 \mathrm{~s}$ for PinSage $(K=2)$ and NGCF $(K=2)$, and about 20s for our proposed LR-GCCF $(K=4)$ on a Ubuntu server with a single GTX 1080Ti. With larger K-th order graph embedding propagations, LR-GCCF costs less time with the linear embedding propagation. The runtime time on the Gowalla dataset for each model is about one third of the time compared to the time cost of the Amazon Books, and the overall trend of the time comparison is similar as analyzed above. 
Table 3: Performance of HR@N and NDCG@N on Amazon Books dataset.

\begin{tabular}{|c|c|c|c|c|c|c|c|c|c|c|}
\hline \multirow{2}{*}{ Models } & \multicolumn{2}{|c|}{$\mathrm{N}=10$} & \multicolumn{2}{|c|}{$\mathrm{N}=20$} & \multicolumn{2}{|c|}{$\mathrm{N}=30$} & \multicolumn{2}{|c|}{$\mathrm{N}=40$} & \multicolumn{2}{|c|}{$\mathrm{N}=50$} \\
\hline & HR & NDCG & HR & NDCG & HR & NDCG & HR & NDCG & HR & NDCG \\
\hline BPR & 0.01851 & 0.01710 & 0.02853 & 0.02169 & 0.03821 & 0.02564 & 0.04737 & 0.02911 & 0.05556 & 0.03205 \\
\hline GC-MC & 0.02063 & 0.01898 & 0.03196 & 0.02408 & 0.04242 & 0.02835 & 0.05226 & 0.03206 & 0.06133 & 0.03532 \\
\hline PinSage & 0.02043 & 0.01872 & 0.03210 & 0.02404 & 0.04298 & 0.02844 & 0.05239 & 0.03199 & 0.06165 & 0.03529 \\
\hline NGCF & 0.02071 & 0.01892 & 0.03244 & 0.02425 & 0.04343 & 0.02872 & 0.05329 & 0.03243 & 0.06263 & 0.03576 \\
\hline$L-G C-M C$ & 0.02092 & 0.01916 & 0.03248 & 0.02443 & 0.04355 & 0.02894 & 0.05394 & 0.03286 & 0.06335 & 0.03623 \\
\hline$R-G C-M C$ & 0.01962 & 0.01796 & 0.03084 & 0.02307 & 0.04153 & 0.02742 & 0.05139 & 0.03115 & 0.06032 & 0.03434 \\
\hline$L-G C C F$ & 0.02067 & 0.01909 & 0.03200 & 0.02424 & 0.04312 & 0.02876 & 0.05310 & 0.03254 & 0.06218 & 0.03579 \\
\hline$L R-G C C F$ & 0.02209 & 0.02040 & 0.03407 & 0.02583 & 0.04532 & 0.03039 & 0.05532 & 0.03416 & 0.06498 & 0.03761 \\
\hline
\end{tabular}

Table 4: Performance of HR@N and NDCG@N on Gowalla dataset.

\begin{tabular}{|l|l|l|l|l|l|l|l|l|l|l|}
\hline \multirow{2}{*}{ Models } & \multicolumn{3}{|c|}{$\mathrm{N}=10$} & \multicolumn{2}{c|}{$\mathrm{N}=20$} & \multicolumn{3}{c|}{$\mathrm{N}=30$} & \multicolumn{3}{c|}{$\mathrm{N}=40$} & \multicolumn{3}{c|}{$\mathrm{N}=50$} \\
\cline { 2 - 12 } & HR & NDCG & HR & NDCG & HR & NDCG & HR & NDCG & HR & NDCG \\
\hline BPR & 0.1041 & 0.1011 & 0.1378 & 0.1126 & 0.1664 & 0.1221 & 0.1908 & 0.1299 & 0.2122 & 0.1365 \\
\hline GC-MC & 0.1042 & 0.1010 & 0.1388 & 0.1127 & 0.1701 & 0.1222 & 0.1969 & 0.1307 & 0.2213 & 0.1381 \\
\hline PinSage & 0.1057 & 0.1042 & 0.1390 & 0.1153 & 0.1682 & 0.1250 & 0.1935 & 0.1330 & 0.2146 & 0.1395 \\
\hline NGCF & 0.1083 & 0.1094 & 0.1403 & 0.1197 & 0.1679 & 0.1288 & 0.1931 & 0.1368 & 0.2142 & 0.1432 \\
\hline$L-G C-M C$ & 0.1045 & 0.1010 & 0.1399 & 0.1132 & 0.1701 & 0.1234 & 0.1957 & 0.1316 & 0.2184 & 0.1386 \\
\hline$R-G C-M C$ & 0.1034 & 0.1000 & 0.1391 & 0.1123 & 0.1690 & 0.1224 & 0.1941 & 0.1305 & 0.2163 & 0.1373 \\
\hline$L-G C C F$ & 0.1044 & 0.1007 & 0.1412 & 0.1135 & 0.1721 & 0.1240 & 0.1977 & 0.1322 & 0.2196 & 0.1390 \\
\hline LR-GCCF & $\mathbf{0 . 1 1 4 8}$ & $\mathbf{0 . 1 1 3 6}$ & $\mathbf{0 . 1 5 1 8}$ & $\mathbf{0 . 1 2 5 9}$ & $\mathbf{0 . 1 8 3 6}$ & $\mathbf{0 . 1 3 6 5}$ & $\mathbf{0 . 2 1 1 3}$ & $\mathbf{0 . 1 4 5 3}$ & $\mathbf{0 . 2 3 5 5}$ & 0.1527 \\
\hline
\end{tabular}

Table 5: Performance of HR@20 and NDCG@20 with different depth K.

\begin{tabular}{|l|l|l|l|l|}
\hline \multirow{2}{*}{ Depth K } & \multicolumn{2}{|c|}{ Amazon Books } & \multicolumn{2}{c|}{ Gowalla } \\
\cline { 2 - 5 } & HR@ 20 & NDCG@20 & HR @ 20 & NDCG@ 20 \\
\hline $\mathrm{K}=0$ & 0.0285 & 0.0217 & 0.1378 & 0.1126 \\
\hline $\mathrm{K}=1$ & 0.0317 & 0.0242 & 0.1504 & 0.1246 \\
\hline $\mathrm{K}=2$ & 0.0327 & 0.0248 & 0.1506 & 0.1248 \\
\hline $\mathrm{K}=3$ & 0.0337 & 0.0255 & $\mathbf{0 . 1 5 1 8}$ & $\mathbf{0 . 1 2 5 9}$ \\
\hline $\mathrm{K}=4$ & $\mathbf{0 . 0 3 4 1}$ & $\mathbf{0 . 0 2 5 8}$ & 0.1494 & 0.1241 \\
\hline $\mathrm{K}=5$ & 0.0340 & 0.0257 & 0.1504 & 0.1247 \\
\hline
\end{tabular}

\section{Detailed Model Analysis}

We would analyze the influence of the recursive label propagation depth $K$, and a detailed analysis of the learned embeddings of the residual preference prediction in LR-GCCF.

Table 5 shows the results on LR-GCCF with different $\mathrm{K}$ values. Particularly, the layer-wise propagation part disappears when $K=0$, i.e., our proposed model degenerates to BPR. As can be observed from Table 5, when $\mathrm{K}$ increase from 0 to 1 , the performance increases quickly on both datasets. For Amazon Books, the best performance reaches with four propagation depth. Meanwhile, our model reaches the best performance when $K=3$ on Gowalla.

In order to better show the effect of residual preference prediction, we design a simplified version of our proposed model that only removes the residual structure in our proposed model. We call the simplified model as L-GCCF. For L-GCCF and LR-GCCF, with each predefined depth $K$, we calculate the cosine similarity of each pair of users (items) between their K-th layer output embedding, i.e., $\mathbf{e}^{K}$ for each node of the graph. The statistics of the mean and variance

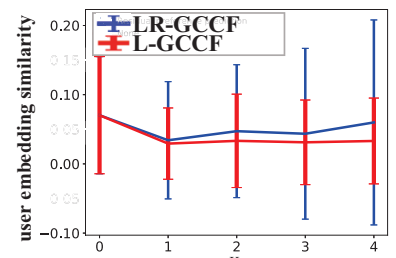

(a) Errorbar of user embedding similarity

\begin{tabular}{|l|l|l|l|l|l|}
\hline Depth & 0 & 1 & 2 & 3 & 4 \\
\hline
\end{tabular}

\begin{tabular}{|l|l|l|l|l|l|l|}
\hline L-GCCF & 0.0217 & 0.0224 & 0.0242 & 0.0242 & 0.0241 \\
\hline
\end{tabular}

\begin{tabular}{|l|l|l|l|l|l|l|}
\hline LR-GCCF & 0.0217 & $\mathbf{0 . 0 2 4 2}$ & $\mathbf{0 . 0 2 4 8}$ & $\mathbf{0 . 0 2 5 5}$ & $\mathbf{0 . 0 2 5 8}$ \\
\hline
\end{tabular}

(c) Overall NDCG@20 on recommendation performance
Figure 3: Comparisons with and without residual preference prediction structure under different layers depth $K$ on Amazon Books dataset.

of user-user (item-item) embedding similarities are shown in Figure 3. It obviously shows our proposed model has larger variance of the user-user cosine similarity compared to its counterparts L-GCCF that does not perform residual learning. This empirically validates that the residual learning could partially alleviate the over smoothing issue, and achieves better performance. Please note that, the overall trend on the Gowalla dataset is similar, and we do not show it due to page limit.

\section{Conclusions}

In this paper, we revisited the current GCN based recommendation models, and proposed a LR-GCCF model for CF 
based recommendation. LR-GCCF was mainly composed of two parts: First, with the recent progress of simple GCNs, we empirically removed the non-linear transformations in GCNs, and replaced it with linear embedding propagations. Second, to reduce the over smoothing effect introduced by higher layers of graph convolutions, we designed a residual preference prediction part with a residual preference learning process at each layer. Extensive experimental results clearly showed the effectiveness and efficiency of our proposed model. In the future, we would like to explore how to better integrate the representations of different layers with well defined deep neural architectures for better enhancing $\mathrm{CF}$ based recommendation.

Acknowledgments. This work was supported in part by grants from the National Key Research and Development Program of China (2018YFB0804205), the National Natural Science Foundation of China (Grant No. 61725203, 61972125, 61602147, 61932009, 61732008, 61722204), and Zhejiang Lab (No.2019KE0AB04).

\section{References}

Berg, R. v. d.; Kipf, T. N.; and Welling, M. 2018. Graph convolutional matrix completion. In KDD Workshop.

Camps-Valls, G.; Marsheva, T. V. B.; and Zhou, D. 2007. Semisupervised graph-based hyperspectral image classification. TGRS 45(10):3044-3054.

Chen, J.; Zhang, H.; He, X.; Nie, L.; Liu, W.; and Chua, T.-S. 2017. Attentive collaborative filtering: Multimedia recommendation with item-and component-level attention. In SIGIR, 335-344.

Cheng, H.-T.; Koc, L.; Harmsen, J.; Shaked, T.; Chandra, T.; Aradhye, H.; Anderson, G.; Corrado, G.; Chai, W.; Ispir, M.; et al. 2016. Wide \& deep learning for recommender systems. In Recsys workshop, 7-10.

Gilmer, J.; Schoenholz, S. S.; Riley, P. F.; Vinyals, O.; and Dahl, G. E. 2017. Neural message passing for quantum chemistry. In ICML, 1263-1272.

Goodfellow, I.; Bengio, Y.; and Courville, A. 2016. Deep learning. MIT press.

Gu, Q.; Zhou, J.; and Ding, C. 2010. Collaborative filtering: Weighted nonnegative matrix factorization incorporating user and item graphs. In SDM, 199-210.

Hamilton, W.; Ying, Z.; and Leskovec, J. 2017. Inductive representation learning on large graphs. In NIPS, 1024-1034.

He, K.; Zhang, X.; Ren, S.; and Sun, J. 2016. Deep residual learning for image recognition. In CVPR, 770-778.

He, X.; Liao, L.; Zhang, H.; Nie, L.; Hu, X.; and Chua, T.-S. 2017. Neural collaborative filtering. In $W W W, 173-182$.

Huang, G.; Liu, Z.; Van Der Maaten, L.; and Weinberger, K. Q. 2017. Densely connected convolutional networks. In CVPR, 47004708.

Huang, Z.; Chung, W.; and Chen, H. 2004. A graph model for e-commerce recommender systems. JASIST 55(3):259-274.

Kipf, T. N., and Welling, M. 2017. Semi-supervised classification with graph convolutional networks. In ICLR.

Klicpera, J.; Bojchevski, A.; and Günnemann, S. 2019. Predict then propagate: Graph neural networks meet personalized pagerank. ICLR.
Koren, Y.; Bell, R.; and Volinsky, C. 2009. Matrix factorization techniques for recommender systems. Computer 42(8):30-37.

Li, D.; Hung, W.-C.; Huang, J.-B.; Wang, S.; Ahuja, N.; and Yang, M.-H. 2016. Unsupervised visual representation learning by graphbased consistent constraints. In ECCV, 678-694.

Li, Q.; Han, Z.; and Wu, X.-M. 2018. Deeper insights into graph convolutional networks for semi-supervised learning. In $A A A I$.

Liang, D.; Charlin, L.; McInerney, J.; and Blei, D. M. 2016. Modeling user exposure in recommendation. In $W W W, 951-961$.

Liu, N. N., and Yang, Q. 2008. Eigenrank: a ranking-oriented approach to collaborative filtering. In SIGIR, 83-90.

Monti, F.; Bronstein, M.; and Bresson, X. 2017. Geometric matrix completion with recurrent multi-graph neural networks. In NIPS, 3697-3707.

Rendle, S.; Freudenthaler, C.; Gantner, Z.; and Schmidt-Thieme, L. 2009. Bpr: Bayesian personalized ranking from implicit feedback. In $U A I, 452-461$.

van den Berg, R.; Kipf, T. N.; and Welling, M. 2017. Graph convolutional matrix completion. $K D D$.

Velickovic, P.; Cucurull, G.; Casanova, A.; Romero, A.; Lio, P.; and Bengio, Y. 2018. Graph attention networks. In ICLR.

Wang, X.; He, X.; Wang, M.; Feng, F.; and Chua, T.-S. 2019. Neural graph collaborative filtering. In SIGIR.

Wu, L.; Liu, Q.; Chen, E.; Yuan, N. J.; Guo, G.; and Xie, X. 2016. Relevance meets coverage: A unified framework to generate diversified recommendations. TIST 7(3):39.

Wu, L.; Ge, Y.; Liu, Q.; Chen, E.; Hong, R.; Du, J.; and Wang, M. 2017. Modeling the evolution of users' preferences and social links in social networking services. TKDE 29(6):1240-1253.

Wu, F.; Zhang, T.; Souza Jr, A. H. d.; Fifty, C.; Yu, T.; and Weinberger, K. Q. 2019a. Simplifying graph convolutional networks. In ICML, 6861-6871.

Wu, L.; Sun, P.; Fu, Y.; Hong, R.; Wang, X.; and Wang, M. 2019b. A neural influence diffusion model for social recommendation. In SIGIR, 235-244.

Wu, Y.; Liu, H.; and Yang, Y. 2018. Graph convolutional matrix completion for bipartite edge prediction. In KDIR, 51-60.

Wu, Z.; Shen, C.; and Van Den Hengel, A. 2019. Wider or deeper: Revisiting the resnet model for visual recognition. Pattern Recognition 90:119-133.

Xu, K.; Li, C.; Tian, Y.; Sonobe, T.; Kawarabayashi, K.-i.; and Jegelka, S. 2018. Representation learning on graphs with jumping knowledge networks. In ICML.

Xu, F.; Lian, J.; Han, Z.; Li, Y.; Xu, Y.; and Xie, X. 2019a. Relation-aware graph convolutional networks for agent-initiated social e-commerce recommendation. In CIKM, 529-538.

Xu, K.; Hu, W.; Leskovec, J.; and Jegelka, S. 2019b. How powerful are graph neural networks? In ICLR.

Ying, R.; He, R.; Chen, K.; Eksombatchai, P.; Hamilton, W. L.; and Leskovec, J. 2018. Graph convolutional neural networks for web-scale recommender systems. In SIGKDD, 974-983.

Zhao, L.; Song, Y.; Zhang, C.; Liu, Y.; Wang, P.; Lin, T.; Deng, M.; and Li, H. 2019. T-gen: A temporal graph convolutional network for traffic prediction. TITS.

Zheng, L.; Lu, C.-T.; Jiang, F.; Zhang, J.; and Yu, P. S. 2018. Spectral collaborative filtering. In RecSys, 311-319.

Zhu, X.; Ghahramani, Z.; and Lafferty, J. D. 2003. Semisupervised learning using gaussian fields and harmonic functions. In $I C M L, 912-919$. 